\title{
Transverse Single-Spin Asymmetries of Midrapidity Eta Mesons at PHENIX
}

\author{
Nicole Lewis *t \\ University of Michigan \\ E-mail: nialewis@umich.edu
}

\begin{abstract}
Transverse Single-Spin Asymmetries (TSSAs) of proton-proton collisions have a long history of revealing the richness of QCD. Large TSSA were originally discovered in fixed target experiments but have been found to persist in collisions up to $\sqrt{s}=510 \mathrm{GeV}$ and transverse momenta up to about $7 \mathrm{GeV}$, well into the perturbative regime of QCD, and yet their origin remains poorly understood. TSSA measurements have allowed for the development of both transverse momentum dependent and collinear twist-3 descriptions of nonperturbative spin-momentum correlations in the nucleon as well as in the process of hadronization. As hadrons, eta mesons are sensitive to both initial- and final-state nonperturbative effects for a mix of parton flavors. Their comparison to neutral pions may provide information on potential effects due to strangeness, isospin, or mass. The status of the TSSA of eta mesons at midrapidity for $200 \mathrm{GeV}$ proton-proton collisions from the PHENIX 2015 data set will be shown.
\end{abstract}

23rd International Spin Physics Symposium - SPIN2018 -

10-14 September, 2018

Ferrara, Italy

\footnotetext{
* Speaker.

${ }^{\dagger}$ On behalf the PHENIX collaboration
} 


\section{Introduction}

Because of asymptotic freedom, perturbative QCD can only be used to calculate high energy partonic scattering cross sections, not the structure or formation of bound states of the strong force. Thus calculations for high energy hadronic scattering require nonperturbative functions to describe how the partons come out of and form bound states, which must be measured from data. Parton Distribution Functions (PDFs) describe the initial-state behavior of partons in the proton and Fragmentation Functions (FFs) describe the process of the scattered partons hadronizing into a particular final state particle. Factorization is the property that the perturbative partonic scattering, the initial-, and the final-state nonperturbative effects can all be expressed in separate functions then convolved together to calculate the entire cross section. This measurement is an update of the PHENIX TSSA of $\eta$ mesons at midrapidity and $\sqrt{s}=200 \mathrm{GeV}$. Because gluons self couple, a variety of leading order partonic processes can contribute to $p p \rightarrow \eta X$. At midrapidity and $\sqrt{s}=200$ $\mathrm{GeV}$, this process is dominated by $g g \rightarrow g g$ and $g g \rightarrow q \bar{q}$ at low $\eta p_{T}$ between about 2 and $4 \mathrm{GeV}$, which corresponds to the lowest bin of this measurement. Mid-range $p_{T}$, about 5 to $20 \mathrm{GeV}$, is dominated by $q g \rightarrow q g$ and $q \bar{q}$ interactions dominate for $p_{T}$ above $20 \mathrm{GeV}$ (well beyond the scope of this measurement) [1].

Hadron transverse single-spin asymmetries study $p^{\uparrow}+p \rightarrow h+X$ collisions and measure the azimuthal asymmetry in the yields of $h$ with the respect to the proton spin direction. In 1978 it was shown that if these asymmetries were only caused by perturbative QCD effects they would be less than one percent [2], but TSSAs have been measured to be as large as $40 \%$ and consistent over a wide range of collision energies [3-8]. Because the perturbative part of the calculation cannot account for the measured large spin-momentum correlations, we must reexamine the nonperturbative part.

Traditional nucleon structure functions are collinear, meaning that one integrates over the internal dynamics of the parton and only considers the momentum of the parton collinear to the direction that the proton travels. Transverse Momentum Dependent (TMD) functions explicitly depend on the internal nonperturbative transverse momentum $k_{T}$ of the partons within the proton in addition to the hard scale $Q$. In order for TMD factorization to apply $k_{T}^{2}<<Q^{2}$ and so in order to ensure that a measurement is within the TMD regime, it must be a two scale process that is sensitive to both $k_{T}$ and $Q$. An example of a TMD-PDF is the Sivers function which is the correlation between the transverse spin of the proton and the nonperturbative transverse momentum of the parton. It is odd under a parity-time transformation (also known as naive-time-reversal-odd) which leads to the predicted sign change between the Drell-Yan and Semi Inclusive Deep Inelastic Scattering (SIDIS) processes [9]. In SIDIS it is not only possible to measure both the soft and hard scales of collisions, but also isolate the effects from particular TMD functions. There is some indication that the Sivers asymmetry might be slightly larger for $K^{+}$mesons than for $\pi^{+}$, which might indicate larger spin-momentum correlations for strange quarks in the proton [10]. An example of a TMD fragmentation function is the Collins function which is the correlation between the transverse spin of a quark and the transverse momentum of the hadron it fragments into. The Collins FF is chiral odd, meaning that in order to preserve chiral symmetry it must be measured in convolution with another chiral odd function, such as in dihadron production in $e^{+} e^{-}$annihilation where nonzero spin-momentum correlations are used to measure the convolution of two Collins 
FFs [11]. In SIDIS the Collins FF can be measured in convolution with the transversity PDF and measurements indicate that spin-momentum correlations are larger for $K^{+}$than $\pi^{+}$in SIDIS [12], again indicating that strangeness might play an interesting role in these nonperturbative effects.

Transverse single-spin asymmetries have been measured to be as large as $40 \%$ for $p^{\uparrow}+p \rightarrow$ $\pi^{0}+X$ for $p_{T}$ as high as $7 \mathrm{GeV}$ [8], well into the perturbative regime of QCD. TSSAs in protonproton collisions uncovered the need for a TMD framework, but unfortunately inclusive particle production in hadronic collisions is not sensitive to the soft scale $k_{T}$ and in general only a single scale hadron $p_{T}$ is available. However, there is an alternative to the TMD framework in the higher twist functions. Traditional PDFs and FFs are twist-2, they only consider interactions with one parton in the bound state at a time. Twist- 3 functions are used to describe the spin-momentum correlations in the proton and in hadronization by calculating the effects due to the quantum mechanical interference of interacting with one parton versus interacting with two partons at the same $x$. These multiparton correlations can be either the quark-gluon-quark (qgq) correlation functions, the quantum interference of scattering off of a quark and a gluon versus a single quark of the same flavor, or three-gluon (ggg) correlation functions, interacting with two gluons versus interacting with one. These twist-3 correlation functions can describe both nucleon structure and hadronization [13]. Twist-3 functions are related to nonperturbative transverse momentum $k_{T}$ moments of twist-2 TMD-PDFs [14] and fragmentation functions, but they do not explicitly depend on $k_{T}$. Thus measurements only need to be dependent on a single scale: $p_{T}$ as a proxy for the hard scale $Q$. Twist-3 spin-momentum correlations have been shown to successfully predict the behavior of TSSA, such as in [15] where the final-state twist-3 fragmentation function was parametized from a forward STAR $A_{N}^{\pi^{0}}$ result at $\sqrt{s}=200 \mathrm{GeV}$ and this function was then evolved and used to predict successfully both the sign and approximate amplitude of the asymmetry at $\sqrt{s}=500 \mathrm{GeV}$.

\section{Analysis}

The Relativistic Heavy Ion Collider (RHIC) is the only collider in the world that can run polarized proton beams, allowing for TSSAs to be measured at high energies. This is made possible by the Siberian snakes that are located $180^{\circ}$ apart on the ring that maintain the polarization in both proton beams for hours at a time. In order to avoid systematic effects, the polarization direction can switch between bunches: up to the sky or down to the ground. PHENIX is one of the large multipurpose detectors located around the RHIC ring. This analysis looks at $\eta \rightarrow \gamma \gamma$ decays and uses data from the central ElectroMagnetic Calorimeter (EMCal) which has an acceptance of $|\eta|<$ 0.35 and two nearly back-to-back arms that each cover $\Delta \phi=\pi / 2$ in azimuth. Six out of eight of the sectors are made of lead scintillator and the other two are made of lead glass and both located on the bottom east side of the detector. This measurement uses the transversely polarized protonproton data set from 2015, which had an integrated luminosity of $60 \mathrm{pb}^{-1}$, and uses a trigger that selects for high energy clusters.

Transverse single-spin asymmetries generally measure the asymmetry as a function of the angle from the polarization direction and then fit a sinusoid in order to measure amplitude. But because of the limited azimuthal acceptance of the PHENIX central arms, midrapidity TSSA measurements at PHENIX generally integrate over both sides of the detector and perform an azimuthal correction: 


$$
A_{N}=\frac{1}{\langle|\cos \phi|\rangle} \frac{1}{P} A_{N}^{r a w}
$$

In this formula $\phi=0$ is at a right angle to proton spin direction where the asymmetry is maximal. The acceptance correction $\langle|\cos \phi|\rangle$ corrects for the dilution from the asymmetry being measured over such a large range in $\phi$. The asymmetry is also diluted by the proton bunches not being $100 \%$ polarized, and so it must be divided by the polarization $P$ of the beam which for this data set was on average $57 \%$.

This result uses the relative luminosity formula that measures the asymmetry in counts for when the beam was polarized up versus down for photon pairs from one side of the EMCal at a time:

$$
A_{N}^{\text {raw }}=\frac{N_{L}^{\uparrow}-R N_{L}^{\downarrow}}{N_{L}^{\uparrow}+R N_{L}^{\downarrow}} .
$$

Here the up and down arrows refer to spin orientation of the beam and the subscript $L$ refers to counts to the left of the polarized beam going direction. There is an equivalent formula for the right side, but the signs in the numerator are flipped to such that the TSSA remains a left-right asymmetry. Because the relative luminosity formula takes the ratio of counts coming from the same detector, effects from detector acceptance and efficiency cancel out. Thus this calculation only needs to be corrected for the relative luminosity of the different beam configurations: $R=L^{\uparrow} / L^{\downarrow}$. It is calculated by using the number of events from bunches that were spin up that fired the minimum bias trigger, divided by the number of events from bunches with polarization direction down that fired the same trigger.

As mentioned before, proton-proton collisions at RHIC have both beams polarized and the polarization direction can be varied bunch to bunch. Thus the asymmetry can be calculated once by keeping track of the polarization direction for only the beam traveling clockwise through the PHENIX detector, effectively averaging over the polarization of the beam traveling to counterclockwise. Then the asymmetry can be recalculated by considering the polarization orientation of the counter-clockwise beam and averaging over the polarization of the clockwise beam. Note these two separate asymmetry measurements are statistically independent and are averaged together for the final result.

\subsection{Systematic Studies}

The square root TSSA formula is used as an additional cross check. For this formula effects from both relative luminosity and detector acceptance and efficiency cancel out to the first order. The differences in the result between this formula and the relative luminosity formula are used as an estimate of the systematic error:

$$
A_{N}^{\text {raw }}=\frac{\sqrt{N_{L}^{\uparrow} N_{R}^{\downarrow}}-\sqrt{N_{L}^{\downarrow} N_{R}^{\uparrow}}}{\sqrt{N_{L}^{\uparrow} N_{R}^{\downarrow}}+\sqrt{N_{L}^{\downarrow} N_{R}^{\uparrow}}} .
$$

Another cross check is to find the $\sin \phi$ modulation. Here the asymmetry is calculated as a function of $\phi_{s}$, the angle between the spin up direction and detected photon pair, and then fit to a 
sine function to measure asymmetry:

$$
A_{N}^{r a w} \sin \left(\phi_{s}\right)=\frac{N^{\uparrow}\left(\phi_{s}\right)-R N^{\downarrow}\left(\phi_{s}\right)}{N^{\uparrow}\left(\phi_{s}\right)+R N^{\downarrow}\left(\phi_{s}\right)} .
$$

Even ignoring PHENIX's limited central acceptance, this is not the optimal way to measure the asymmetry given that this result is consistent with zero and statistically limited. It does serve as a good cross check however, since it essentially averages over the PHENIX central arms and does the azimuthal correction at the same time.

Bunch shuffling is used as a way of quantifying the systematic error present in the sample. The variance of the measured TSSAs was consistent with statistical fluctuations for all $p_{T}$ bins but the lowest, where there was some indication of an additional systematic uncertainty which was assigned. We note that the lowest $p_{T}$ bin has the highest background. An additional systematic error was assigned based on how the background fraction changed with varying the fit region on the diphoton invariant mass distributions.

\subsection{Background Correction}

When looking at an invariant mass distribution like the one shown in Fig. 1, even if there is a clear resonance peak, it is impossible to tell which photon pairs within this peak region came from an $\eta \rightarrow \gamma \gamma$ decay and which came from the combinatorial background. Thus the asymmetry of this background must be measured and subtracted off from the peak asymmetry:

$$
A_{N}^{S}=\frac{A_{N}^{S+B}-r A_{N}^{B}}{1-r}
$$

where $S$ stands for signal and $B$ stands for background and $r=N^{B} /\left(N^{B}+N^{S}\right)$ is the background fraction, the fraction of photon pairs within the invariant mass peak that come from the combinatorial background. The $A_{N}^{S+B}$ asymmetry is calculated with photon pairs with invariant mass $480<M_{\gamma \gamma}<620 \mathrm{MeV}$ (the blue region in Fig. 1) and the $A_{N}^{B}$ asymmetry is calculated using photon pairs with invariant mass $300<M_{\gamma \gamma}<400 \mathrm{MeV}$ or $700<M_{\gamma \gamma}<800 \mathrm{MeV}$ (the red regions in Fig. 1). These background regions are close enough to the signal range such that they approximate the behavior of the background within that peak, but far enough away such that $\eta \rightarrow \gamma \gamma$ decays do not contribute to the background asymmetry. The background fraction $r$ is estimated by doing a fit to the invariant mass spectrum, using a gaussian for the signal and a third degree polynomial to approximate the background. The background fraction is calculated as a function of $p_{T}$ and also separately for each side of the EMCal.

\section{Results}

Fig. 2 shows this new result plotted with the older PHENIX midrapidity TSSA $\eta$ meson result from 2012 data [16]. This updated result is a factor of about 3 to 4 increase in precision. Fig. 3 is this same updated result but with a smaller range on the $y$-axis. It shows that the asymmetry is consistent with zero to within 0.005 at low $p_{T}$, but it may indicate some hint of a trend. More data will be needed to draw more concrete conclusions. 


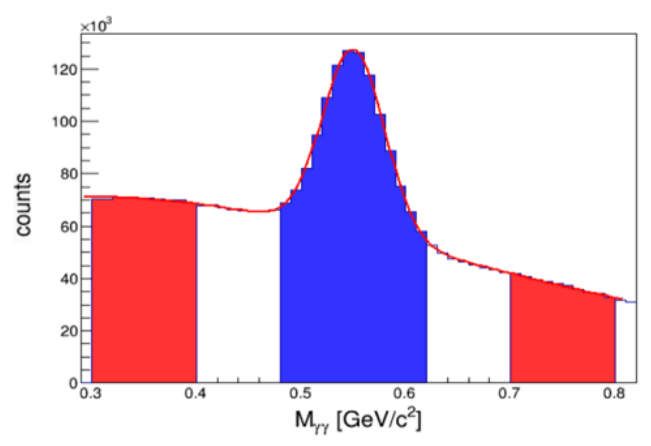

Figure 1: Example invariant mass histogram for photon pairs in the West Arm with $4<p_{T}<5 \mathrm{GeV}$.

$\pi^{0}$ and $\eta$ mesons are both flavorless, pseudoscalars that can decay into two photons. $\eta \rightarrow \gamma \gamma$ in general yields much more statistically limited measurements than $\pi^{0} \rightarrow \gamma \gamma$ because the branching ratio is lower and due to the mass difference there are more pions than $\eta$ mesons produced. But given the difference in quark composition: $\pi^{0}=\frac{1}{\sqrt{2}}(u \bar{u}-d \bar{d})$ and $\eta=\frac{1}{\sqrt{3}}(u \bar{u}+d \bar{d}+s \bar{s})$, differences seen in $A_{N}^{\pi^{0}}$ and $A_{N}^{\eta}$ can provide insight into the role that strangeness and isospin play in fragmentation. Also since the $\eta$ meson is four times heavier than the $\pi^{0}$, differences in $\pi^{0}$ and $\eta$ results could also point to the role of hadron mass in nonperturbative final state effects. PHENIX's most recent midrapidity results showed that $A_{N}^{\pi^{0}}$ was consistent with zero to within $10^{-4}$ at low $p_{T}[17]$.

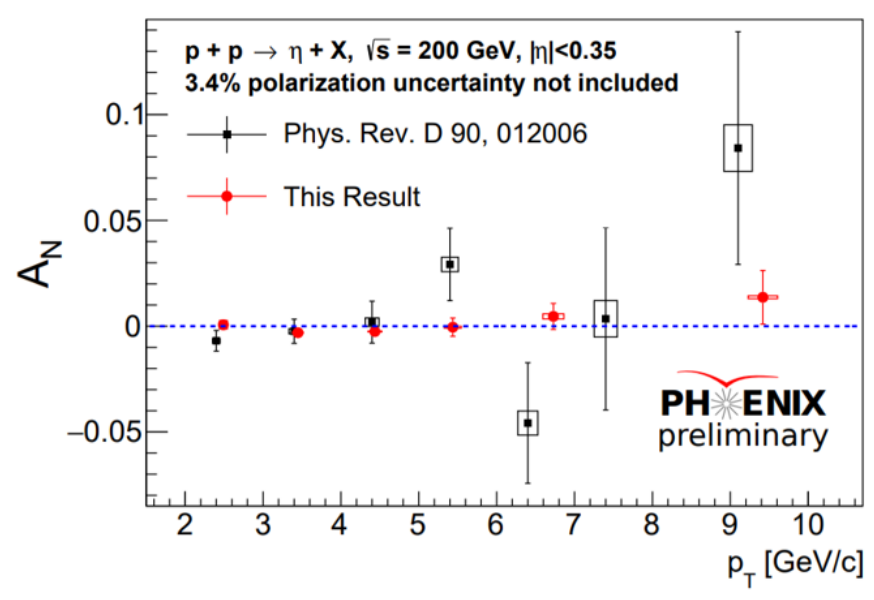

Figure 2: TSSA of midradpity $\eta$ mesons at $\sqrt{s}=200 \mathrm{GeV}$ plotted with the previous PHENIX result.

\section{Conclusion}

Transverse single-spin asymmetries probe parton dynamics in both initial- and final-state effects. Traditional twist- 2 collinear nonperturbative functions cannot account for the large spinmomentum correlations that have been observed in prior experiments. Transverse momentum dependent functions study the effect of initial-state partons and final-state hadrons having some 


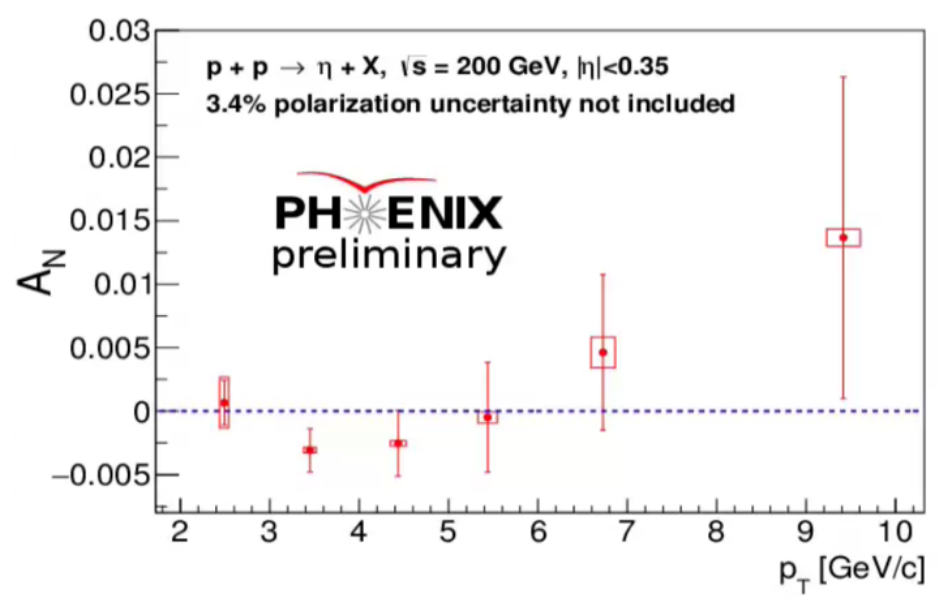

Figure 3: TSSA of midradpity $\eta$ mesons at $\sqrt{s}=200 \mathrm{GeV}$ from PHENIX 2015 data.

nontrivial, soft-scale transverse momentum. Twist-3 collinear functions study the quantum interference between having only one active parton per bound state versus having two and can be easily compared to measurements that are only sensitive to a single hard scale energy. This document shows the new $\eta A_{N}$ PHENIX result at midrapidity and $\sqrt{s}=200 \mathrm{GeV}$. It is consistent with zero and improves the precision by a factor of about 3 to 4 when compared with the previous PHENIX result. It will help constrain twist-3 collinear functions and is sensitive to the impact of strangeness on these functions.

\section{References}

[1] A. Adare et al. (PHENIX Collaboration), Cross section and double helicity asymmetry for $\eta$ mesons and their comparison to $\pi^{0}$ production in $p+p$ collisions at $\sqrt{s}=200 \mathrm{GeV}$, Phys. Rev. D 83 (2011) 032001 [hep-ex/1009.6224].

[2] G. L. Kane, J. Pumplin, and W. Repko, Transverse Quark Polarization in Large- $p_{T}$ Reactions, $e^{+} e^{-}$ Jets, and Leptoproduction: A Test of Quantum Chromodynamics, Phys. Rev. Lett. 41 (1978) 1689.

[3] R. D. Klem, et al., Measurement of Asymmetries of Inclusive Pion Production in Proton-Proton Interactions at 6 and $11.8 \mathrm{GeV} / \mathrm{c}$, Phys. Rev. Lett. 36 (1976) 929.

[4] D. L. Adams et al. (FNAL E704 Collaboration), Analyzing power in inclusive $\pi^{+}$and $\pi^{-}$production at high $x_{F}$ with a $200 \mathrm{GeV}$ polarized proton beam, Phys. Lett. B 264 (1991) 462.

[5] C. E. Allgower et al. (E925 Collaboration), Measurement of analyzing powers of $\pi^{+}$and $\pi^{-}$ produced on a hydrogen and a carbon target with a 22-GeV/c incident polarized proton beam, Phys. Rev. D 65 (2002) 092008.

[6] I. Arsene et al. (BRAHMS Collaboration), Single-Transverse-Spin Asymmetries of Identified Charged Hadrons in Polarized pp Collisions at $\sqrt{s}=62.4 \mathrm{GeV}$, Phys. Rev. Lett. 101 (2008) 042001 [nucl-ex/0801.1078].

[7] B. I. Abelev et al. (STAR Collaboration), Forward Neutral-Pion Transverse Single-Spin Asymmetries in $p+p$ Collisions at $\sqrt{(s)}=200 \mathrm{GeV}$, Phys. Rev. Lett. 101 (2008) 222001 [hep-ex/0801.2990]. 
[8] Y. Pan for the STAR Collaboration, Transverse Single Spin Asymmetries of Forward $\pi^{0}$ and Jet-like Events in $\sqrt{s}=500 \mathrm{GeV}$ Polarized Proton Collisions at STAR, in proceedings of The 21st International Symposium on Spin Physics, I. J. of Mod. Phys: Conf. Ser. 40 (2008) 1660037.

[9] J. C. Collins, Leading-twist single-transverse-spin asymmetries: Drell-Yan and deep-inelastic scattering, Phys. Lett. B 536 (2002) 43 [hep-ph/0204004].

[10] A. Airapetian et al. (HERMES Collaboration), Observation of the Naive-T-Odd Sivers Effect in Deep-Inelastic Scattering, Phys. Rev. Lett. 103 (2009) 15002 [hep-ex/0906.3918].

[11] R. Seidl et al. (Belle Collaboration), Measurement of azimuthal asymmetries in inclusive production of hadron pairs in $e^{+} e^{-}$annihilation at $\sqrt{s}=10.58$ Gev, Phys. Rev. Lett. (2008) 78032011 [hep-ex/0805.2975]. Erratum: PRD 86 (2012) 039905.

[12] C. Adolph et al.(COMPASS Collaboration), Collins and Sivers asymmetries in muonproduction of pions and kaons off transversely polarised protons, Phys. Lett. B 744 (2015) 250 [hep-ex/1408.4405].

[13] D. Pitonyak, Transverse spin observables in hard-scattering hadronic processes within collinear factorization, Int. J. of Mod. Phys. A 31 (2016) 1630049 [hep-ph/1608.05353].

[14] X. Ji, Qiu, J., Vogelsang, W., \& Yuan, F., Unified Picture for Single Transverse-Spin Asymmetries in Hard-Scattering Processes, Phys. Rev. Lett. 97 (2006) 082002 [hep-ph/0602239].

[15] K. Kanazawa, Y. Koike, A. Metz, and D. Pitonyak, Towards an explanation of transverse single-spin asymmetries in proton-proton collisions: The role of fragmentation in collinear factorization, Phys. Rev. D 89 (2014) 111501(R) [hep-ph/1404.1033].

[16] A. Adare et al. (PHENIX Collaboration), Measurement of transverse-single-spin asymmetries for midrapidity and forward-rapidity production of hadrons in polarized $p+p$ collisions at $\sqrt{s}=200$ and 62.4 GeV, Phys. Rev. D 90 (2014) 012006 [hep-ex/1312.1995].

[17] N. Novitzky, Transverse single asymmetries in $\pi^{0}$ production in $p+p, p+A l$, and $p+A u$ collisions at mid-rapidity using the PHENIX detector system, presentation, 22nd International Spin Symposium (2016) Urbana-Champaign, IL, USA. 